\title{
High Energy Neutrino Flash from Far-UV/X-ray Flares of Gamma-Ray Bursts
}

\author{
Kohta Murase ${ }^{1 *}$ and Shigehiro Nagataki ${ }^{1,2}$ 计 \\ ${ }^{1}$ Yukawa Institute for Theoretical Physics, Kyoto University, \\ Oiwake-cho, Kitashirakawa, Sakyo-ku, Kyoto, 606-8502, Japan \\ ${ }^{2}$ KIPAC, Stanford University, P.O.Box 20450, MS 29, Stanford, CA, 94309, USA
}

(Dated: April 20, 2006)

\begin{abstract}
The recent observations of bright optical and X-ray flares by the Swift satellite suggest these are produced by the late activities of the central engine. We study the neutrino emission from far-UV/X-ray flares under the late internal shock model. Since the efficiency of pion production in the highest energy is higher than that of the prompt bursts, such neutrino flashes from flares can give comparable or larger contributions to a diffuse very high energy neutrino background if the total energy input into flares is comparable to the radiated energy of the prompt bursts. These signals are very important because they have possibility to probe the nature of flares (baryonic or magnetic, the photon field, the magnetic field, and so on).
\end{abstract}

PACS numbers: 95.85.Ry, 98.70.Rz, 25.20.-x, 14.60.Lm

\section{INTRODUCTION}

High energy neutrino emission from gamma-ray bursts (GRBs) have been expected in the context of the standard internal-external scenario of GRBs. Especially, since the prediction of Waxman \& Bahcall [1], neutrino bursts in the internal shock model have been studied by several authors [2, 3, 4, 5] and neutrino afterglows in the external shock model have also been discussed [6, 7, 8] (see also 9$]$ ).

The standard model of GRBs has succeeded in explaining of many observations, but there had been a few outstanding questions in the study of GRBs before the launch of the Swift satellite (see reviews e.g., 10, 11]). The Swift satellite, which is ideal mission to answer these questions, has presented indeed very fruitful results during the first several months of its operation. Especially in the X-ray band, Swift XRT detected an X-ray afterglow for essentially every burst, which showed the surprising behaviors that are not straightforwardly expected in the pre-Swift era [12]. The early afterglow lightcurve has several surprising features such as an early steep decay, a follow-up shallower-than-normal decay, and one or more X-ray flares and so on (see e.g., [12, 13]). Especially, observations show that many bursts have large $\mathrm{X}$ ray flares superimposed on the underlying afterglow. In the early afterglows of XRF 050406 and GRB 050502b, XRT detected mysterious strong X-ray flares [14, 15, 16], and some flares such as GRB 050607 and GRB 050904 have multiple flares [17, 18]. These observational results suggest the existence of additional emission in the early afterglow phase besides the conventional forward shock emission.

In this letter we calculate high energy neutrino emission from FUV/X-ray flares under the late internal shock

\footnotetext{
*Electronic address: kmurase@yukawa.kyoto-u.ac.jp

${ }^{\dagger}$ Electronic address: nagataki@yukawa.kyoto-u.ac.jp
}

model. Our method of calculation using GEANT4 [19] is the same as in Murase \& Nagataki [5], but quantitatively improved 20, 21]. Now, large neutrino detectors such as IceCube 22], ANTARES 23], NESTOR 24] are being constructed. In near future, these detectors may detect high energy neutrino signals correlated with flares.

\section{THE MODEL}

The flares typically happen hundreds of seconds after the trigger of prompt emissions or earlier. In some cases, they occur around a day after the burst. The observed typical time scale is larger than that of the prompt emission, which is $\delta t \sim(10-100)$ s $[13,14]$. The typical collision radius is expressed by commonly used relation, $r \approx 10^{14.5}(\Gamma / 30)^{2}(\delta t / 6 \mathrm{~s}) \mathrm{cm}$. The amplitudes of the flares are usually larger than the underlying afterglow component by a factor of several, but can be much larger. These can be even comparable to or higher than the prompt burst component which is typically $L_{\mathrm{X}}^{\mathrm{GRB}} \sim\left(10^{49}-10^{52}\right)$ ergs/s around $(1-10) \mathrm{keV}$ band. Hereafter we take $L_{\max }=\left(10^{48}-10^{51}\right) \mathrm{ergs} / \mathrm{s}$ as a peak luminosity of FUV/X-ray flares. Although some flares allow for the possibility of external shock processes, the general features of the flares suggest that this phenomenon is best interpreted as a late internal central engine activity [12]. The variability of some GRB afterglows implies that the engine may last much longer than the duration of the bursts 26], although a possible mechanism for re-activity of the central engine is unknown [27, 28, 29]. We suppose the late internal shock occurs a few minutes after the prompt $\gamma$-ray emission, powering a new unsteady relativistic outflow. Falcone et al. shows that the case of GRB 050502b implies the late outflow has the smaller Lorentz factor 15]. Hence, we assume that the typical Lorentz factors of the ejected material are smaller than the prompt emission, setting $\Gamma_{\mathrm{s}} \sim 10$ and $\Gamma_{\mathrm{f}} \sim 100$ as the typical Lorentz factors of the slow and fast shells respectively. We can estimate 


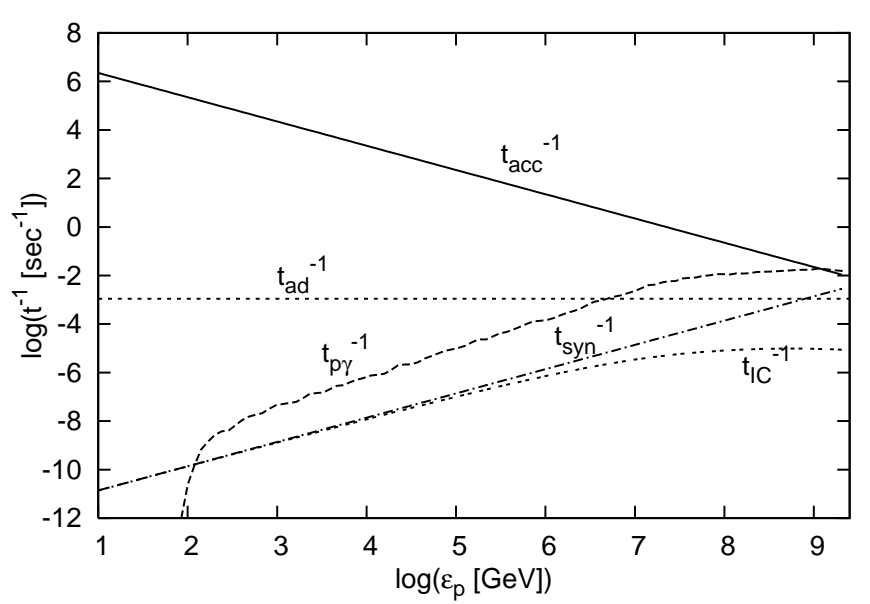

FIG. 1: Various cooling time scales and the acceleration time scale for FUV-ray flares with $L_{\max }=10^{49} \mathrm{ergs} / \mathrm{s}$ and $\xi_{B}=$ 1. Note that energy scale is measured in the shell comoving frame.

the Lorentz factor of the merged shell $\Gamma \approx \sqrt{\Gamma_{\mathrm{f}} \Gamma_{\mathrm{s}}} \simeq 30$, and the Lorentz factor of the internal shocks can be estimated by $\Gamma_{\mathrm{sh}} \approx\left(\sqrt{\Gamma_{\mathrm{f}} / \Gamma_{\mathrm{s}}}+\sqrt{\Gamma_{\mathrm{s}} / \Gamma_{\mathrm{f}}}\right) / 2 \sim$ a few. This re-generated internal shocks are called as the late internal shocks [30]. Actually the exact radiation mechanism producing the flares is unclear. Now, to see typical parameters, we assume that flares are produced by the synchrotron emission. The minimal Lorentz factor of electrons is estimated by $\gamma_{e, \mathrm{~m}} \approx \epsilon_{e}\left(m_{p} / m_{e}\right)\left(\Gamma_{\mathrm{sh}}-1\right)$. Since we can estimate the intensity of magnetic field by $B=7.7 \times 10^{3} \mathrm{G}_{B}^{1 / 2}\left(\Gamma_{\mathrm{sh}}\left(\Gamma_{\mathrm{sh}}-1\right) / 2\right)^{1 / 2} L_{\mathrm{M}, 50}^{1 / 2} \Gamma_{30}^{-1} r_{14.5}^{-1}$, the observed break energy is, $E^{\mathrm{b}}=\gamma_{e, \mathrm{~m}}^{2} \Gamma e B / m_{e} c \sim$ $0.1 \mathrm{keV} \epsilon_{e,-1}^{2} \epsilon_{B,-1}^{1 / 2}\left(\Gamma_{\mathrm{sh}}-1\right)^{5 / 2}\left(\Gamma_{\mathrm{sh}} / 2\right)^{1 / 2} L_{\mathrm{M}, 50}^{1 / 2} \Gamma_{30}^{-1} r_{14.5}^{-1}$, where $L_{\mathrm{M}}$ is the outflow luminosity. Therefore, the typical emitted energy is in the soft X-ray band.

For numerical calculations, we simply set the break energy and adopt the power-law spectrum similarly with that of the case of the prompt emission. Although most of flares are actually well fitted by a Band function or cutoff power-law model [15], this treatment does not change our results so much. We use the following expression in the comoving frame, $d n / d \varepsilon=n_{\mathrm{b}}\left(\varepsilon / \varepsilon^{\mathrm{b}}\right)^{-\alpha}$ for $\varepsilon^{\min }<\varepsilon<\varepsilon^{\mathrm{b}}$ or $d n / d \varepsilon=n_{\mathrm{b}}\left(\varepsilon / \varepsilon^{\mathrm{b}}\right)^{-\beta}$ for $\varepsilon^{\mathrm{b}}<\varepsilon<\varepsilon^{\max }$, where we set $\varepsilon^{\min }=0.1 \mathrm{eV}$ because the synchrotron self-absorption will be crucial below this energy [31] and $\varepsilon^{\max }=1 \mathrm{MeV}$ because the pair absorption will be crucial above this energy [32]. Actually we do not know the peak energy and the lower spectral index of many flares 33. So we allow for the existence of far-ultraviolet-ray (FUV-ray) flares and take $\varepsilon^{\mathrm{b}}=(0.01-0.1) \mathrm{keV}$ in the comoving frame. We assume $\alpha=1$ and set $\beta=2.2$ [13] similarly with the prompt emission. The photon energy density is $U_{\gamma}=\int d \varepsilon \varepsilon d n / d \varepsilon$. The magnetic energy density and the nonthermal proton energy density are expressed by $U_{B}=\xi_{B} U_{\gamma}$ and $U_{p}=\xi_{\text {acc }} U_{\gamma}$ respectively. Although we have done wide parameter surveys, we show the one case

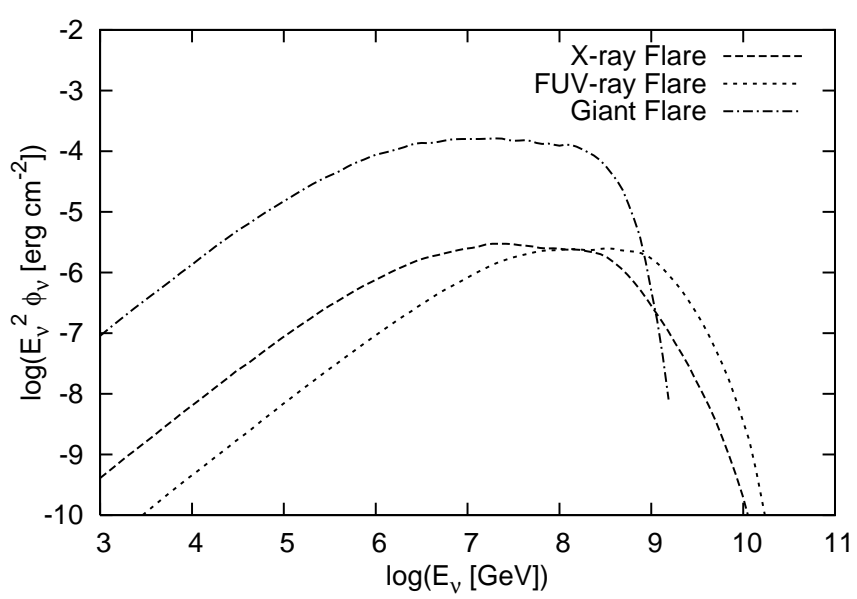

FIG. 2: The observed muon-neutrino $\left(\nu_{\mu}+\bar{\nu}_{\mu}\right)$ spectra for one GRB event at $z=0.1$. X-ray Flare: $L_{\max }=10^{49} \mathrm{ergs} / \mathrm{s}$, $\xi_{B}=1, \xi_{\text {acc }}=10$, and $N=1$. FUV-ray Flare: $L_{\max }=$ $10^{48} \mathrm{ergs} / \mathrm{s}, \xi_{B}=1, \xi_{\text {acc }}=10$, and $N=10$. Giant Flare (FUV-ray flare): $L_{\max }=10^{50} \mathrm{ergs} / \mathrm{s}, \xi_{B}=0.1, \xi_{\text {acc }}=30$, and $N=1$.

where $r=8.1 \times 10^{14} \mathrm{~cm}$ and $\Delta \approx\left(r / 2 \Gamma^{2}\right)=4.5 \times 10^{11}$ $\mathrm{cm}$, according to $\delta t=30 \mathrm{~s}$ at $z=1$.

To obtain the pion production rate and estimate the maximal energy of accelerated protons, we need to take into account following various cooling time scales. We consider, the synchrotron cooling time written by $t_{\mathrm{syn}}=$ $3 m_{p}^{4} c^{3} / 4 \sigma_{\mathrm{T}} m_{e}^{2} \varepsilon_{p} U_{B}$, the inverse-Compton (IC) cooling time which is given by Jones [34], the adiabatic cooling time comparable to the dynamical time, and the photomeson cooling time which is evaluated by,

$$
t_{p \gamma}^{-1}\left(\varepsilon_{p}\right)=\frac{c}{2 \gamma_{p}^{2}} \int_{\bar{\varepsilon}_{\mathrm{th}}}^{\infty} d \bar{\varepsilon} \sigma_{p \gamma}(\bar{\varepsilon}) \kappa_{p}(\bar{\varepsilon}) \bar{\varepsilon} \int_{\bar{\varepsilon} / 2 \gamma_{p}}^{\infty} d \varepsilon \varepsilon^{-2} \frac{d n}{d \varepsilon}
$$

where $\bar{\varepsilon}$ is the photon energy in the rest frame of proton, $\gamma_{p}$ is the proton's Lorentz factor, $\kappa_{p}$ is the inelasticity of proton, and $\bar{\varepsilon}_{\text {th }}$ is the threshold photon energy for photomeson production in the rest frame of the incident proton, which is $\bar{\varepsilon}_{\text {th }} \approx 145 \mathrm{MeV}$. From various time scales, we can estimate the total cooling time scale by $t_{p}^{-1} \equiv t_{p \gamma}^{-1}+t_{\mathrm{syn}}^{-1}+t_{\mathrm{IC}}^{-1}+t_{\mathrm{ad}}^{-1}$. We believe that not only electrons but also protons can be accelerated by the first-order Fermi acceleration mechanism and assume $d n_{p} / d \varepsilon_{p} \propto \varepsilon_{p}^{-2}$. By the condition $t_{\mathrm{acc}}<t_{p}$, we can estimate the maximal energy of accelerated protons. Here, we take the acceleration time scale by $t_{\text {acc }} \sim \varepsilon_{p} / e B c$ optimistically. We also set the minimal energy of protons to $10 \mathrm{GeV}$ because this will be around $\sim \Gamma_{\mathrm{sh}} m_{p} c^{2}$. One of numerical results is shown in Fig. 1.

Here, we evaluate $f_{p \gamma} \equiv t_{\text {dyn }} / t_{p \gamma}$ by $\Delta$-resonance approximation. After performing the second integral in Eq. (11), we can approximate by,

$$
t_{p \gamma}^{-1} \simeq \frac{U_{\gamma}}{2 \varepsilon^{\mathrm{b}}} c \sigma_{\Delta} \kappa_{p}\left(\bar{\varepsilon}_{\Delta}\right) \frac{\Delta \bar{\varepsilon}}{\bar{\varepsilon}_{\Delta}}\left\{\begin{array}{l}
\left(\bar{\varepsilon}_{\Delta} / 2 \gamma_{p} \varepsilon^{\mathrm{b}}\right)^{-(\beta-1)} \\
\left(\bar{\varepsilon}_{\Delta} / 2 \gamma_{p} \varepsilon^{\mathrm{b}}\right)^{-(\alpha-1)}
\end{array}\right.
$$




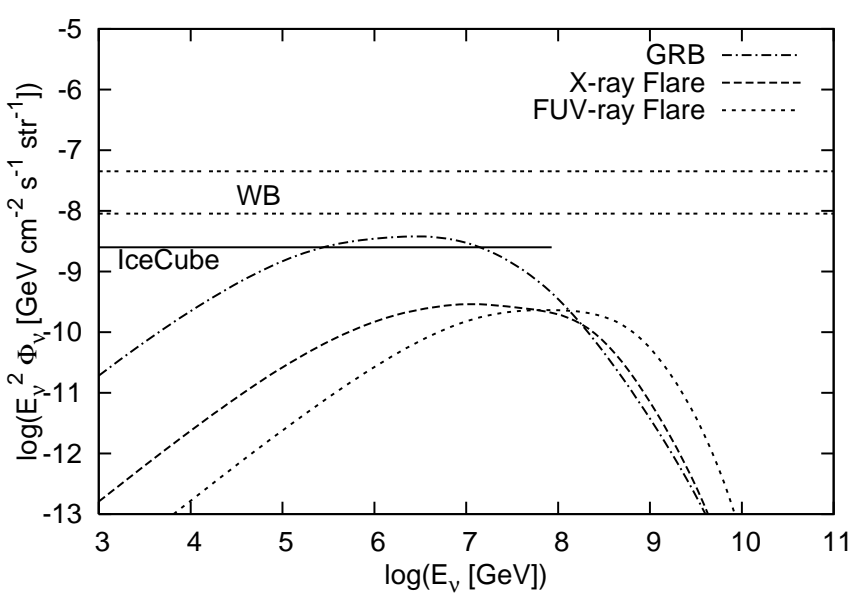

FIG. 3: The neutrino background from GRBs for $f_{\mathrm{F}} \xi_{\mathrm{acc}}=$ 0.5. X-ray Flare: $L_{\max }=10^{49} \mathrm{ergs} / \mathrm{s}$ and $\xi_{B}=1$. FUVray Flare: $L_{\max }=10^{48} \mathrm{ergs} / \mathrm{s}$ and $\xi_{B}=1$. GRB: taken from [5] with $E_{\gamma, \mathrm{sh}}^{\text {iso }}=2 \times 10^{51} \mathrm{ergs}, \xi_{B}=1, \xi_{\text {acc }}=10$, and $r=\left(10^{13}-10^{14.5}\right) \mathrm{cm}$. WB: Waxman-Bahcall bounds [1].

where $\sigma_{\Delta} \sim 5 \times 10^{-28} \mathrm{~cm}^{2}, \kappa_{p}\left(\bar{\varepsilon}_{\Delta}\right) \sim 0.2$, and $\bar{\varepsilon}_{\Delta} \sim 0.3$ $\mathrm{GeV}$, and $\Delta \bar{\varepsilon} \sim 0.2 \mathrm{GeV}[1]$. Here, we have included the effect of multipion production and high inelasticity which is moderately important, and multiplied by a factor of $\sim(2-3)$ in Eq. (2) [5]. Hence, we can obtain,

$$
f_{p \gamma} \simeq 10 \frac{L_{\max , 49}}{r_{14.5} \Gamma_{30}^{2} E_{\mathrm{keV}}^{\mathrm{b}}}\left\{\begin{array}{cc}
\left(E_{p} / E_{p}^{\mathrm{b}}\right)^{\beta-1} & \left(E_{p}<E_{p}^{\mathrm{b}}\right) \\
\left(E_{p} / E_{p}^{\mathrm{b}}\right)^{\alpha-1} & \left(E_{p}^{\mathrm{b}}<E_{p}\right)
\end{array}\right.
$$

where $E_{p}^{\mathrm{b}} \simeq 0.5 \bar{\varepsilon}_{\Delta} m_{p} c^{2}$ is the proton break energy. From Eq. (31), we can conclude that a significant fraction of high energy accelerated protons cannot escape the source without photomeson productions. In the case of bright X-ray flares whose luminosity is larger than $10^{49} \mathrm{ergs} / \mathrm{s}$ and FUV-ray flares, almost all protons accelerated to the very high energy region will be depleted.

\section{RESULTS AND DISCUSSIONS}

Even in the case of the prompt emission, we can not expect neutrino events from one burst unless the source is nearby or energetic. Similarly, we can expect high energy neutrino flashes from one burst only in the case of nearby or energetic flares. In Fig. 2, we show an example of the observed neutrino flux from the source at $z=0.1$. The expected muon events for above TeV energy neutrinos are $N_{\mu}=0.02$ events in the case of X-ray Flare in Fig. 2. We can expect $N_{\mu}=1.4$ events when the flare is energetic and much nonthermally baryonic. Note that, in the case of an energetic flare, the maximal neutrino energy becomes small because the highest energy protons suffer from the photomeson cooling very much.

We can estimate a diffuse neutrino background from FUV/X-ray flares for specific parameter sets under the

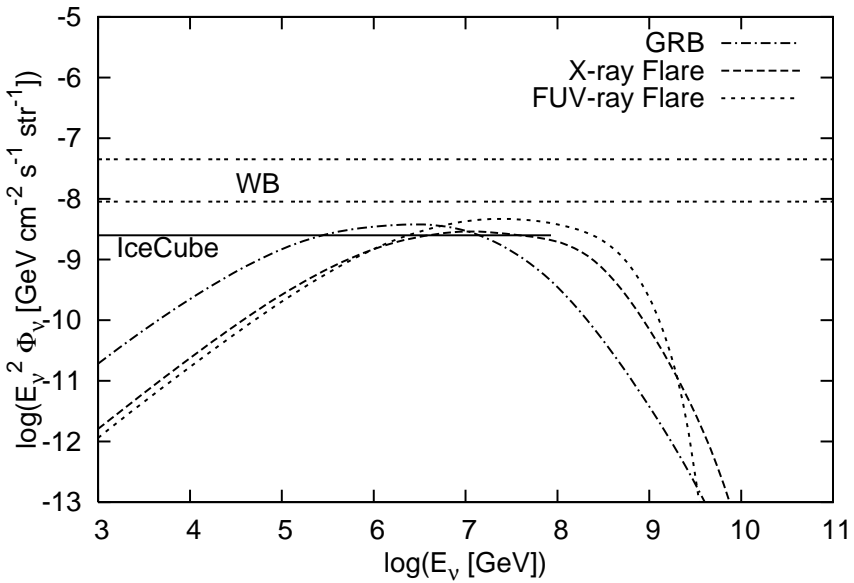

FIG. 4: The neutrino background from GRBs for $f_{\mathrm{F}} \xi_{\text {acc }}=5$. X-ray Flare: $L_{\max }=10^{49} \mathrm{ergs} / \mathrm{s}$ and $\xi_{B}=1$. FUV-ray Flare: $L_{\max }=10^{49} \mathrm{ergs} / \mathrm{s}$ and $\xi_{B}=0.1$. GRB: taken from [5] with $E_{\gamma, \mathrm{sh}}^{\text {iso }}=2 \times 10^{51} \mathrm{ergs}, \xi_{B}=1, \xi_{\text {acc }}=10$, and $r=\left(10^{13}-\right.$ $\left.10^{14.5}\right) \mathrm{cm}$. WB: Waxman-Bahcall bounds [1].

standard $\Lambda$ CDM cosmology $\left(\Omega_{\mathrm{m}}=0.3, \Omega_{\Lambda}=0.7 ; H_{0}=\right.$ $\left.71 \mathrm{~km} \mathrm{~s}^{-1} \mathrm{Mpc}^{-1}\right)$. Assuming that the long GRB rate traces the starformation rate (SFR), we shall use the SF3 model of Porciani \& Madau [35] combined with the normalization of overall GRB rates obtained by Guetta et al., [36] and we set $z_{\max }=11$ since the re-ionization is likely to occur within $z \sim(7-13)$ 37]. Even if the GRB rate traces metallicity, but this will not change our results so much. X-ray flares are detected in at least $(1 / 3$ - 1/2) of Swift GRBs 13]. So we can expect that flares are common in GRBs. Here, introducing the ratio of the energy emitted by flares to that of the prompt emission, $f_{\mathrm{F}} \equiv E_{\text {flare }} / E_{\mathrm{GRB}}$, we estimate neutrino flux analytically. First, we can express the total number spectrum of accelerated protons, using $\varepsilon_{p, \max } \sim 10^{9} \mathrm{GeV}$,

$$
E_{p}^{2} \frac{d N_{p}}{d E_{p}} \simeq 1.6 \times 10^{50} N f_{\mathrm{b}} \xi_{\text {acc }, 10} L_{\max , 49}\left(\frac{r_{14.5}}{\Gamma_{30}^{2}}\right) \mathrm{erg}
$$

where $f_{\mathrm{b}}$ is the beaming factor and $N$ is the number of flares. Since we have already estimated $f_{p \gamma}$ by Eq. (3), by replacing $2.5 N f_{\mathrm{b}} L_{\max } r / \Gamma^{2} c$ with the total emitted energy from flares $E_{\text {flare }}$, we can estimate the diffuse neutrino background as follows,

$$
\begin{aligned}
E_{\nu}^{2} \frac{d F_{\nu}}{d E_{\nu} d \Omega} & \sim \frac{c}{4 \pi H_{0}} \frac{1}{4} \min \left[1, f_{p \gamma}\right] E_{p}^{2} \frac{d N_{p}}{d E_{p}} R_{\mathrm{GRB}}(0) f_{z} \\
& \simeq 3 \times 10^{-9} \mathrm{GeVcm}^{-2} \mathrm{~s}^{-1} \mathrm{str}^{-1} \\
& \times\left(\frac{f_{\mathrm{F}} \xi_{\mathrm{acc}}}{5}\right)\left(\frac{f_{z}}{3}\right) E_{\mathrm{GRB}, 51} \min \left[1, f_{p \gamma}\right]
\end{aligned}
$$

where $f_{z}$ is the correction factor for the possible contribution from high redshift sources. $f_{\mathrm{F}} \xi_{\text {acc }}$ expresses the ratio of the nonthermal baryon energy of flares to the prompt radiated energy. If it is comparable to $E_{\mathrm{GRB}}$, we can expect a significant contribution to a neutrino background 
from flares. In this letter, we take $f_{\mathrm{F}} \xi_{\text {acc }}=(0.5-5)$. Our numerical results obtained by the same method as in our previous paper are shown in Fig. 3 and Fig. 4. For example in Fig. 4, we can obtain $N_{\mu}=7.6$ events/yr and $N_{\mu}=8.8$ events/yr respectively. Although these values on above $\mathrm{TeV}$ energy neutrinos are smaller than that of the prompt emission (shown by GRB in Fig. 3 and Fig. 4), $N_{\mu}=21$ events/yr [5], neutrino signals from flares dominate and exceed those from prompt emissions in the very high energy region above a few $\mathrm{PeV}$ range. These signals may be detected by IceCube and/or Auger which can provide information on the nature of flares. For example, while the energy of neutrinos from flares can be very high unless the magnetic field is too low, too copious photon field will reduce the maximum proton energy and the following neutrino energy. Hence, the detection of signals can give us information on not only the magnetic field but also the photon field. Moreover, such neutrino detection may include signals from FUV-ray flares, that are not detected due to absorption by neutral hydrogen both in host galaxy and in our Galaxy. Combined with GLAST mission which may detect sub-GeV flashes by IC [33] or $\pi^{0}$ decay, these high energy neutrino emission may be important as a probe of FUV-ray emissions. We expect such neutrino flashes from flares should be in coincidence with the early afterglow phase. Especially, some of events will be correlated with observed flares.

Many flares are likely to be interpreted as the late activity of the central engine. Such an energy injection is also one of common interpretations for early flattening of the X-ray afterglow, which is evident for a good fraction of X-ray afterglows. The injected energy will be comparable to that of the prompt burst [12]. These late activity may supply the extra nonthermal baryons. Furthermore, we do not know the total radiated energy itself by flares at present. The fluences of some X-ray flares are smaller than that of the bursts, but can be even comparable to or higher than that of the bursts. There are also some GRBs which have multiple flares. Since we do not know the peak energy of most flares, there might be a significant fraction of the FUV flares. Moreover, we do not know the opening angle of such late outflows themselves. In the case of the prompt emission, the typical value of the beaming factor expressed by $E_{\mathrm{GRB}}=f_{\mathrm{b}} E_{\gamma, \text { tot }}^{\text {iso }}$, is $f_{\mathrm{b}} \sim 0.01$. The late slow outflow might have the larger opening angles than the prompt fast outflow, because $\theta_{\min }=1 / \Gamma$ of the late outflows is larger than that of the initial outflows. These unknown features of flares will be unveiled through more multiwave-length observations by Swift, GLAST and so on. We expect future neutrino observations will also give us some information on the physical parameters of flares, such as $f_{\mathrm{F}} \xi_{\text {acc }}$.

Not only long GRBs but also short GRBs also may accompany flares [38]. Since short GRBs are closer than long GRBs, we can expect a significant contribution from FUV/X-ray flares of short GRBs if such flares are baryonic origins rather than magnetic origins.

Waxman \& Bahcall [1] predicted neutrino burst under the assumption that GRBs are the main sources of ultra-high-energy cosmic rays (UHECRs). In the case of the prompt bursts, the optical thickness for the photomeson production can be smaller than the unity especially at larger radii $r \gtrsim 10^{14}\left(E_{\gamma, \mathrm{sh}}^{\mathrm{iso}} / 10^{51} \mathrm{ergs}\right)^{1 / 2} \mathrm{~cm}$ and the UHECRs can be produced in such regions. In flares, it is more difficult to generate UHECRs especially in the case of FUV flares since the optical thickness for the photomeson production is larger. Of course, if we assume the larger nonthermal baryon loading factor, which is enough large to explain UHECRs in the prompt internal shocks, neutrino events from flares will also be more expectable.

In this letter we do not consider the distribution of parameters of flares, which is still unknown. In both the prompt and the late internal shocks, it is important to know this distribution. It is necessary to get more information by future observations in order to achieve more realistic predictions. So far, we have not taken account of neutrino oscillations. Since we have considered many decaying modes, the production ratio of high energy $\nu_{\mu}$ and $\nu_{e}$ is not 2:1 exactly. But, the neutrinos will be almost equally distributed among flavors as a result of vacuum neutrino oscillations [1]. So there may be a possibility that we can detect $\nu_{\tau}$ through double bang events [39].

\section{Acknowledgments}

K.M. thanks K. Ioka for a lot of profitable suggestions. We also thank K. Toma for introducing the recent topics to us. S.N. is partially supported by Grants-in-Aid for Scientific Research from the Ministry of Education, Culture, Sports, Science and Technology of Japan through No. 14102004, 14079202, and 16740134.
[1] E. Waxman and J. Bahcall, Phys. Rev. Lett., 78, 2292 (1997)

[2] C.D. Dermer and A. Atoyan, Phys. Rev. Lett., 91, 071102 (2003)

[3] D. Guetta, D.W. Hooper, J. Alvarez-Muñiz, F. Halzen, and E. Reuveni, Astropart. Phys., 20, 429 (2004)

[4] K. Asano, ApJ, 623. 967 (2005)

[5] K. Murase and S. Nagataki, Phys. Rev. D, 73, 063002
(2006)

[6] E. Waxman and J. Bahcall, ApJ, 541, 707 (2000)

[7] Z.G. Dai and T. Lu, ApJ, 551, 249 (2001)

[8] Z. Li, Z.G. Dai, and T. Lu, A\&A, 396, 303 (2002)

[9] C.D. Dermer, ApJ, 574, 65 (2002)

[10] T. Piran, Rev. Mod. Phys., 76, 1143 (2005)

[11] B. Zhang and P. Mészáros, IJMP.A, 19, 2385 (2004)

[12] B. Zhang et al., ApJ, in press, astro-ph/0508321 (2005) 
[13] P.T. O'Brien et al., ApJ, submitted, astro-ph/0601125 (2006)

[14] D.N. Burrows et al., Science, 309, 16 (2005)

[15] A.D. Falcone et al., ApJ, 641, 1010 (2006)

[16] P. Romano et al., A\&A, 450, 59 (2006)

[17] C. Pagani et al., ApJ, accepted, astro-ph/0603658 (2006)

[18] G. Cusumano et al., Nature, 440, 164 (2006)

[19] S. Agostinelli et al., Nuclear Instruments and Methods in Physics Research A, 506, 250-303 (2003), http://wwwasd.web.cern.ch/wwwasd/geant4/geant4.html

[20] Particle Data Group, http://pdg.lbl.gov/

[21] S. Schadmand, Eur. Phys. J. A, 18, 405 (2003)

[22] J. Ahrens et al., Astropart. Phys., 20, 507 (2004)

[23] E. Aslanides et al., Report CPPM-P-1999-02 (1999)

[24] P.K.F. Grieder et al., Nuovo Cimento, 24 C, 771 (2001)

[25] S. Kobayashi et al., ApJ, submitted, astro-ph/0506157 (2005)

[26] K. Ioka, S. Kobayashi and B. Zhang, ApJ, 631, 429 (2005)

[27] D. Proga and M. Begelman, ApJ, 592, 767 (2003)
[28] A. King et al., ApJ, 630, L113 (2005)

[29] R. Perna, P.J. Armitage, and B. Zhang, ApJ, 636, L29 (2006)

[30] Y.Z. Fan and D.M. Wei, MNRAS, 364, L42 (2006)

[31] Z. Li and L.M. Song, ApJ, 608, L17 (2004)

[32] K. Asano and F. Takahara, PASJ, 55, 433 (2003)

[33] Y.Z. Fan and T. Piran, MNRAS, accepted, astro-ph/0601619 (2006)

[34] F.C. Jones, Phys. Rev., 137, 1306 (1965)

[35] C. Porciani and P. Madau, ApJ, 548, 522 (2001)

[36] D. Guetta, T. Piran, and E. Waxman, ApJ, 619, 412 (2005)

[37] D.N. Spergel et al., ApJ, submitted, astro-ph/0603449 (2006)

[38] S. Barthelmy et al., Nature, 438, 994 (2005).

[39] H. Athar, G. Parente, and E. Zas, Phys. Rev. D, 62, 093010 (2000) 